CASE STUDY

\title{
EFFICACY OF MUSIC THERAPY FOR PROMOTING SOCIABILITY IN A CHILD WITH AUTISM SPECTRUM DISORDER
}

\begin{abstract}
A twenty two year old individual, showing deficits in the emotional regulation and socially-apt behavior was referred for this case study by his special educator. The Sociability Domain of the Autism Treatment Evaluation Checklist (ATEC) was used pre and post the intervention. The intervention was based on the Guided Focus Program designed as part of music therapy at Karachi Vocational Training Centre for Intellectually Challenged. The intervention phase lasted for 3 months (36 sessions and each session was of 45 minutes). The participant showed significant changes in the sociability domain of Autism Treatment Evaluation Checklist (ATEC). This case contributes to the evidence base for the efficacy of Music Therapy. However, further research is needed to confirm its long term efficiency while comparing it to other alternative treatments.
\end{abstract}

\section{KEYWORDS}

Asperger's Disorder Autism Spectrum Disorder, Music Therapy, Vocational Training, Anxiety.
Maria Waris Nawaz

Research Psychologist

Vocational Rehabilitation

Karachi Vocational Training Centre for Intellectually Challenged

maria.waris@kvtc.org.uk

\section{Imran Sohail}

Curriculum Consultant

Vocational Rehabilitation

Karachi Vocational Training Centre for

Intellectually Challenged

imran.sohail@kvtc.org.uk

\section{Sana Ayaz}

Principal

Karachi Vocational Training Centre for Intellectually Challenged

sana.ayaz@kvtc.org.uk

[Nawaz MW, Sohail I, Ayaz S. Efficacy of Music Therapy for promoting sociability in a child with Autism Spectrum Disorder: A Single Case Study ( 13 - 16 years) of Karachi. Pak. j. rehabil. 2020;9(2):66-69]

DOI:10.36283/pjr.zU.9.2/013 


\section{INTRODUCTION}

Autism Spectrum Disorder (ASD) is a neurodevelopmental disorder that may have persistent deficits in domains like social interaction, social communication, repetitive behaviors, activities or interests ${ }^{1}$. The symptoms of Autism manifest during early childhood when the child is expected to meet his developmental milestones. Number of studies concluded that music therapy has been an effective treatment approach for individuals with ASD2. However, various interventions of music therapy work differently on autistic individuals; despite it leads to improvisation in sociability among them ${ }^{3}$. Moreover, according to the humanistic perspective and creative music therapy, the Nordoff-Robbins Creative Music Therapy model serves as the base of this research (Figure-1). This model aims at showing response and the sensitivity possessed to music by individuals living with disabilities and illness ${ }^{4}$.

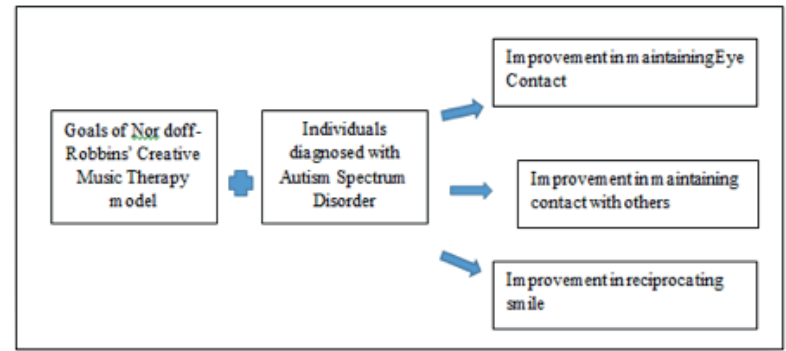

Figure 1: Conceptual framework explaining the use of Music theropy to improve the Sociability domain in individuals diagnsed with ASD.

Figure.1 Nor doff-Robbins' Creative Music Therapy model

\section{CASE}

$\mathrm{N}$ is a twenty two year old boy, who lives in a nuclear family setting, belonging from an Urdu speaking, upper middle class background. He has one elder brother, who is physically and psychologically healthy. N's mother had a normal delivery, without any complications. They moved to South Africa when $\mathrm{N}$ was 2 years old and it was then when they realized with the help of N's teachers and other acquaintances that $\mathrm{N}$ did not respond like other normal children. $N$ was recommended for Neuro-developmental assessments and later was diagnosed with Asperger's Disorder ${ }^{5}$.

$\mathrm{N}$ studied in inclusive education based school in South Africa after his diagnoses for 5 years and later he and his family moved to Karachi, Pakistan. After coming back, his Parents realized that $\mathrm{N}$ faced language barrier, as he was previously able to understand, respond and try to speak English and French in South Africa. But, after returning to Pakistan, most of his same age peers were restricted to be fluent in speaking Urdu Language. This was a setback for $\mathrm{N}$ as he already faced mild social and communicative deficits but now after moving to different country and due to the language barrier, he faced more issues in making himself understood as well as responds to others. This created a lot of irritability, impatience and disruptive behaviors in him due to which he could not maintain healthy relationships with his peers by not trying to maintain direct eye contact, or reciprocate smiles to maintain friendly contact with others even when prompted.

In 2017, N was given admission in Karachi Vocational Training Center for Intellectually Challenged (KVTC). He was mostly seen anxious with a lack of focus and attention; he was easily annoyed and had repetitive hand movements. Hence, in February' 2019, $\mathrm{N}$ was introduced to the Music therapy Unit at KVTC.

\section{METHODOLOGY}

\section{STUDY DESIGN}

A single case study.

\section{STUDY SETTING}

Karachi Vocational Training Centre for intellectually challenged (KVTC).

\section{INCLUSION AND EXCLUSION CRITERIA}

Participant has behavioral issues due to ASD diagnosed by the Psychological Unit of KVTC within the last two years with no physical disability.

\section{INSTRUMENT/TOOL}

Autism Treatment Evaluation Checklist (ATEC) developed by Bernard Rimland and Stephen M. Edelson of the Autism Research Institute was used as a screener in this research consists of 4 subtests:

The sociability domain of Autism Treatment Evaluation Checklist (ATEC) was used in this research pre and post intervention was introduced to the participant to check if there was any difference in his social deficits ${ }^{6}$.

\section{INTERVENTION}

The intervention was the guided focus program music therapy, which helped the participant follow the music therapist's instructions and guidance while maintaining focus when playing the musical instrument. The musical instruments selected for him were a mouth keyboard piano organ which he had to play after the drum beat was played by the therapist. The beats were in a rhythm of 2 hits first, then 4 hits and then 6 hits. Then these were reversed. The participant had to play his instruments to match the rhythmic beats after they were hit on the drum. Apart from that, he was paired with another trainee (diagnosed with Down Syndrome) to have musical Conversation, in which they sat facing each other while taking turns and were prompted to maintain eye contact. Then, they were made to play the high/low game in which on low notes played by the therapist, the participant 
played a different instrument while on high notes he was asked to pick up another instrument. Prompts were given to him between the shifts and he also wore Ear defenders at some occasions when sound became overwhelming for him.

\section{DURATION}

The intervention phase lasted for 3 months (36 sessions over 3 months and each session was of 45 minutes).

\section{RESULTS}

The participant showed significant changes in the sociability domain of Autism Treatment Evaluation Checklist (ATEC) as the scores for the Statements: No Eye Contact, Avoids contact with others and Rarely Smiles changed significantly after the intervention was given (Table-1).

\begin{tabular}{|c|c|c|}
\hline \multicolumn{2}{|c|}{ Table-1 shoes pre-post ATEC checklist } \\
\hline $\begin{array}{c}\text { Pretest } \\
\text { ATEC }\end{array}$ & $\begin{array}{c}\text { Post-test } \\
\text { ATEC }\end{array}$ \\
\hline No Eye Contact & $\checkmark$ & 1 \\
\hline $\begin{array}{c}\text { Avoids contact } \\
\text { with others }\end{array}$ & $\checkmark$ & 1 \\
\hline Rarely Smiles & $\checkmark$ & $\times$ \\
\hline Total & 6 & 2 \\
\hline \multicolumn{3}{|c}{ DISCUSSION }
\end{tabular}

Music therapy has been practiced from ancient times, and carried importance for treating psychological and physical characteristics of disequilibrium occurring in the body ${ }^{7}$. The present study concluded the efficacy of music as therapy that may help in reducing the social deficits of an autistic individual. The results supported the hypothesis of this research, which suggested that the Post-intervention Autism Treatment Evaluation Checklist (ATEC) scores of the participant would be higher as compared to the pre-intervention Autism Treatment Evaluation Checklist (ATEC) scores and similarly the results have been in line with other previous researches as well. According to the Nor doff-Robbins' Creative Music Therapy Model, a collaboration is developed between the therapist and the client, which in this case was supported as well since the participant took an active part in establishing the needed collaboration which helped the therapist to create a timely and an effective treatment plan. In this case study, the participant initially faced extreme irritability when asked to maintain friendly contact with others, even when spoken in English with him during his training hours at KVTC. This was found to be very challenging for his trainers and special educators as he did not reciprocate most of the socially welcoming gestures like smile, hand shake or a wave. Since, KVTC is a vocational training center for the Intellectually Challenged individuals so all of the multi-disciplinary plans followed for every trainee either of academic or of any functional skill are individualized. For N, his plan also followed the same course but initially he was asked to play music in individual sessions with the therapist, then in a pair and then also in a group with other trainees to sit around him while he took his session. Introducing $\mathrm{N}$ to Music therapy was itself challenging for the institution because, according to Islamic beliefs and Pakistani culture, Music has not been fully supported for being used as a therapy or for healing purpose, although in the western culture, music therapy is being used for Autistic individuals along with other advanced tools like robotics ${ }^{8}$. On the basis of the cultural and religious norms, many people believe that music should be forbidden?, and thus the idea of using music as therapy for healing has faced challenges over the past few years, as many mixed beliefs have emerged just when considering the idea of using instruments for producing any kind of music for therapy ${ }^{10}$. According to the results, during the pre-intervention phase, the participant showed no response to three of the major behaviors present in the sociability domain while in the post-intervention phase, the participant was able to successfully maintain eye contact, maintain interaction which was observed by his interactions with the janitor of the center which was like a milestone to be achieved at the start as he has had the least interaction with the janitorial staff of KVTC in the past, although he still faced some resistance in reciprocating smile. Considering this, it can be said that normally it is very challenging for practitioners to change any kind of behaviors in Autistic individuals within a period of 3 months but the results of this case show the changes and proves that Music Therapy is an effective intervention to help autistic individuals deal with their emotional issues. Thus, the aim of using his previous understanding with current intervention helped researchers to gage him in the here and now principle so that he is able to develop control and the concept of time while working with his social deficits, music therapy also helped him in developing synchronous relationship with his cognition and coordination responses indirectly ${ }^{11}$.

\section{CONCLUSION}

The results concluded that music therapy is effective for the patient to overcome some of his sociability deficits around his peers.

\section{REFERENCE}

[1] Diagnostic and Statistical Manual of Mental Disorders: DSM-5. 5th ed., American Psychiatric Association 2018; DSM-V.

[2] Broder-Fingert S, Feinberg E, Silverstein $M$. Music therapy for children with autism spec- 
trum disorder. Jama. 2017;318(6):523-4.

[3] Robbins PN. Creative Music Therapy: Individualized Treatment for the Handicapped Child.1977.

[4] Geretsegger M, Holck U, Bieleninik $Ł$, Gold C. Feasibility of a trial on improvisational music therapy for children with autism spectrum disorder. J. Music Ther. 2016;53(2):93-120.

[5] Cooper R. Diagnostic and statistical manual of mental disorders (DSM). Ko Knowledge Organization. 2018;44(8):668-76.

[6] Geier DA, Kern JK, Geier MR. A comparison of the Autism Treatment Evaluation Checklist (ATEC) and the Childhood Autism Rating Scale (CARS) for the quantitative evaluation of autism. J Ment Health Res Intellect Disabil 2013 ;6(4):255-67.

[7] Yinger OS, Gooding L. Music therapy and music medicine for children and adolescents. Child and Adolescent Psychiatric Clinics 2014;23(3):535-53.

[8] Beer JM, Boren M, Liles KR. Robot assisted music therapy a case study with children diagnosed with autism. In 201611 th ACM/IEEE International Conference on Human-Robot Interaction (HRI) 2016 (pp. 419-420). IEEE.

[9] Sharda M, Tuerk C, Chowdhury R, Jamey K, Foster N, Custo-Blanch M, Tan M, Nadig A, Hyde K. Music improves social communication and brain connectivity outcomes in children with autism: A randomized controlled trial 2018.

[10] NRMT A. US-trained music therapists from East Asian countries found personal therapy during training helpful but when cultural disconnects occur these can be problematic: a qualitative phenomenological study. The Arts in Psychotherapy 2017;55:54-63.

[11] Kashefimehr B, Kayihan H, Huri M. The effect of sensory integration therapy on occupational performance in children with autism. OTJR: occupation, participation and health. 2018;38(2):75-83. 